\title{
Paediatric mental and physical health presentations to emergency departments, Victoria, 2008-15
}

\author{
Harriet Hiscock ${ }^{1}$, Rachel J Neely ${ }^{2}$, Shaoke Lei ${ }^{1,2}$, Gary Freed ${ }^{3}$
}

The known Mental health disorders in children and adolescents are common, but their impact on presentations to emergency departments (EDs) is unknown.

The new From 2008-09 to 2014-15, mental health presentations increased by $6.5 \%$ per year. Rates of presentation with self-harm or stress-related, mood, and behavioural and emotional disorders increased markedly. The burden on ED resources by presentation was greater for mental health than for physical health presentations.

The implications The number of children presenting to EDs with mental health problems is rising. The reasons should be determined so that mental health care for young people can be improved.

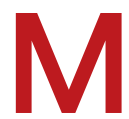
ental health and substance use disorders are the leading cause of disability in children and young adults worldwide. ${ }^{1}$ Over a 12 month period, $14 \%$ of $4-17$-year-olds in Australia — 580000 children and adolescents — are experiencing mental health problems. ${ }^{2}$ Mental health disorders during childhood have adverse effects throughout life, ${ }^{3,4}$ and the onset of $50 \%$ of all mental disorders occurs before the age of 14 years.

Australian children receive mental health care from a variety of community-based organisations, ${ }^{6}$ but it has been anecdotally reported that an increasing number of children and young people are presenting to emergency departments (EDs) with mental health problems. This is worrying; while EDs are equipped to help children who self-harm or take drug overdoses, they are usually noisy, stimulating environments, not conducive to calming agitated patients. ${ }^{7}$ Further, patients who require mental health care can disturb the routine and flow of the ED, and can place a greater demand on resources than medical or trauma patients. ${ }^{7}$ Specialised screening tools and mental health consultants trained in paediatric medicine can reduce the likelihood of hospitalisation and the length of stay in the ED, and also ease security problems, ${ }^{8}$ but they are not available in all EDs. 8,9

Two Australian studies have assessed presentations to EDs by children for mental health problems; both were undertaken more than ten years ago and were single site, cross-sectional studies in tertiary level paediatric EDs. An audit during 2002-03 found that children with psychological emergencies accounted for $0.5 \%$ of all presentations over a 10-month period, and that they were more likely to be admitted to hospital than other ED patients. ${ }^{10} \mathrm{~A}$ retrospective review in another ED over the same period identified 203 adolescents aged 12-18 years with mental health problems, $47 \%$ of whom were admitted to hospital. ${ }^{11}$ A national study in the United States found that the number of ED visits for mental health problems by children aged $10-14$ years increased by $21 \%$ during

\section{Abstract}

Objectives: To identify trends in presentations to Victorian emergency departments (EDs) by children and adolescents for mental and physical health problems; to determine patient characteristics associated with these presentations; to assess the relative clinical burdens of mental and physical health presentations.

Design: Secondary analysis of Victorian Emergency Minimum Dataset (VEMD) data.

Participants, setting: Children and young people, 0-19 years, who presented to public EDs in Victoria, 2008-09 to 2014-15.

Main outcome measures: Absolute numbers and proportions of mental and physical health presentations; types of mental health diagnoses; patient and clinical characteristics associated with mental and physical health presentations.

Results: Between 2008-09 and 2014-15, the number of mental health presentations increased by $6.5 \%$ per year, that of physical health presentations by $2.1 \%$ per year; the proportion of mental health presentations rose from $1.7 \%$ to $2.2 \%$.

Self-harm accounted for $22.5 \%$ of mental health presentations (11 770 presentations) and psychoactive substance use for 22.3\% (11 694 presentations); stress-related, mood, and behavioural and emotional disorders together accounted for 40.3\% (21 127 presentations). The rates of presentations for self-harm, stress-related, mood, and behavioural and emotional disorders each increased markedly over the study period. Patients presenting with mental health problems were more likely than those with physical health problems to be triaged as urgent (2014-15: 66\% v 40\%), present outside business hours (36\% v 20\%), stay longer in the ED (65\% v 82\% met the National Emergency Access Target), and be admitted to hospital (24\% v 18\%).

Conclusions: The number of children who presented to Victorian public hospital EDs for mental health problems increased during 2008-2015, particularly for self-harm, depression, and behavioural disorders.

2006-2011, with a 34\% increase for substance-related disorders and a $71 \%$ rise for impulse control disorders. ${ }^{12}$

Mental health problems may place a greater burden on EDs than physical health presentations in terms of triage category, length of stay, proportion meeting the National Emergency Access Target (NEAT) of being admitted or discharged within 4 hours, ${ }^{13}$ and admission rates. A multi-site study in the US found that paediatric patients with mental health problems were up to three times more likely to be admitted to hospital than patients of the same age with physical problems, ${ }^{14}$ while another multi-site study found that they were more likely to stay in the ED longer. ${ }^{15}$

The questions of whether mental health presentations are increasing in number and pose a greater burden than physical 
health presentations have important policy, service delivery, and workforce training implications. We therefore aimed to document the numbers and proportions of presentations to EDs in Victoria during a 7-year period by patients aged 19 years or younger for mental and physical health problems; the types of mental health diagnoses they received; patient characteristics associated with mental and physical health presentations; and the relative clinical burdens of mental and physical health presentations, including triage category, length of stay, time of presentation, and disposal patterns.

\section{Methods}

Data were obtained from the Victorian Emergency Minimum Dataset $^{16}$ (VEMD) for the financial years 2008-09 to 2014-15. The VEMD is a standardised state dataset comprising de-identified demographic, administrative, and clinical data for presentations to Victorian public hospitals with 24-hour EDs. However, diagnostic codes are usually entered by clinicians who have limited training in coding, which can compromise the diagnostic accuracy of the dataset. ${ }^{17}$

Variables obtained from the VEMD included presentation data (eg, length of stay), departure status (eg, admission), demographic data (eg, age, sex), and diagnosis (full list: online Appendix). Data were collected for children and adolescents aged 0-19 years who presented to general or children's hospitals; it was assumed that young people with mental health problems would not have visited specialty hospitals (eg, maternity hospitals). Hospital campus data were coded by VEMD as metropolitan or rural in a manner that prevented identification of individual patients.

\section{Statistical analyses}

We calculated the absolute number of mental and physical health presentations by children and adolescents to Victorian EDs for each 12-month period. As it was possible that shifts in the age and sex distributions of the general population contributed to changes in ED presentation numbers, we examined annual trends in population growth for Victoria, by VEMD age band and sex, using Australian Bureau of Statistics data for the 7 years assessed. ${ }^{18}$

Mental health presentations were defined as those leading to an F group diagnosis (F00-F99, Mental and behavioural disorders) according to the International Classification of Diseases, revision 10, Australian modification ${ }^{19}$ (ICD-10-AM) or a diagnosis of intentional self-harm. As there is no ICD-10-AM diagnostic code for self-harm, we identified these cases by a primary diagnosis of any physical injury together with coding of human intent equal to intentional self-harm. Differences in the numbers of mental and physical health presentations between the first and last years of the study period were expressed as percentages.

We transformed the Statistical Local Area score in the VEMD to a quintile on the Socio-Economic Index for Areas (SEIFA) - Index of Relative Socio-Economic Advantage and Disadvantage (IRSAD). ${ }^{20}$ The IRSAD is an index of economic and social conditions of people and households in an area, based on census data; a lower score corresponds to greater disadvantage. ${ }^{21}$

We compared patient and presentation characteristics associated with mental and physical health presentations for each financial year. The independence of categorical variables was assessed in $\chi^{2}$ tests. All data were analysed in $\mathrm{R}$ 3.3.2 (R Foundation for Statistical Computing).

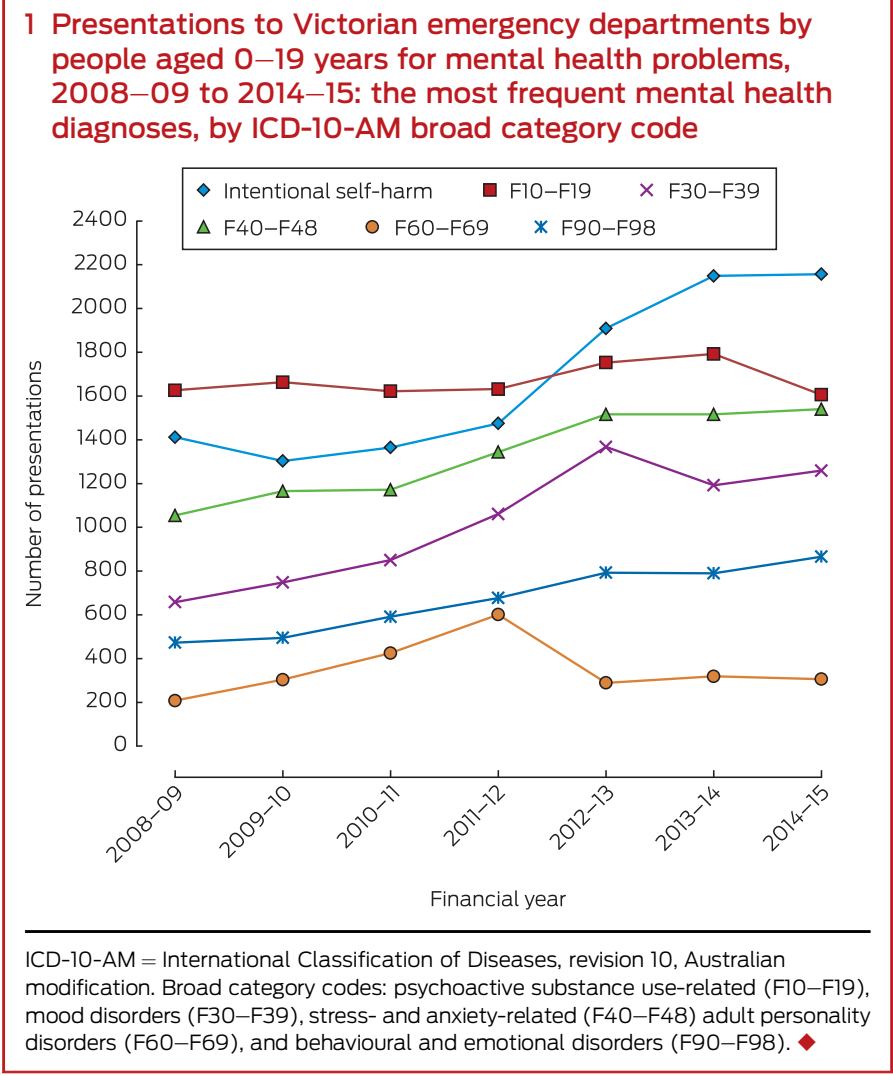

\section{Ethics approval}

The study was screened and approved by the Royal Children's Hospital Human Research Ethics Committee, and was exempted from formal ethics approval. The study was also approved by the Department of Health and Human Services as custodians of the VEMD data.

\section{Results}

Over the 7 years, there were 2763139 presentations to EDs in Victoria by children aged 0-19 years. We excluded 216372 records because they did not include a primary diagnosis; 2546767 presentations were analysed, of which 52359 (2.1\%) were for mental health problems and 2494408 (97.9\%) for physical health problems.

The annual number of mental health presentations increased by $46 \%$, from 5988 in 2008-09 to 8726 in 2014-15 (average annual increase, $6.5 \%$ ). The annual number of physical health presentations grew by 13\%, from 336546 to 381667 (average annual increase, $2.1 \%$ ). The proportion of mental health presentations rose from $1.7 \%$ in $2008-09$ to $2.2 \%$ in $2014-15$.

\section{Mental health diagnoses}

During the 7-year period, 11770 presentations $(22.5 \%$ of all mental health presentations) were for intentional self-harm. The number of presentations for intentional self-harm increased by $52.8 \%$, from 1412 in $2008-09$ to 2157 in 2014-15, becoming the most frequent mental health-related reason for presentation (Box 1).

Mental health problems related to psychoactive substance use (ICD-10-AM codes F10-F19) comprised the second largest category of presentation (11 694 presentations, 22.3\%). Stress and anxiety (ICD-10-AM codes F40-F48), mood disorders (F30-F39), 
2 Characteristics of people aged 0-19 years who presented to Victorian emergency departments, 2008-09 to 2014-15, for mental or physical health problems

2008-09

\section{Outcome}

Presentations (proportion of all

presentations)

Hospital campus (proportion of all presentations)

Metropolitan

Rural

Age band (years)

0-4

5-9

$10-14$

15-19

Sex (boys)

IRSAD quintile,

median (IQR)

Presentation time

08:00-18:00

(in-hours)

18:00-22:00

(after hours)

22:00-02:00

(midnight)

02:00-08:00

(early morning)

Triage category

1-3 (potentially to

immediately life-threatening)

4 (potentially serious) or

5 (less urgent)

Time to treatment ( $\mathrm{min}$ ),

median (IQR)

Length of stay (h)

$<4$

4-11

$12-23$

$>24$

Departure status

Return to usual

residence

Ward at this hospital

Transfer to another

hospital

Departure before

treatment complete

$837 \quad 25035$

(5.4\%) (0.9\%)

\begin{tabular}{cc}
\hline Mental & Physical \\
\hline 5988 & 336546 \\
$(1.7 \%)$ & $(98.3 \%)$ \\
& \\
& \\
4048 & 224465 \\
$(1.8 \%)$ & $(98.2 \%)$ \\
1940 & 112081 \\
$(1.7 \%)$ & $(98.3 \%)$
\end{tabular}

16460958

(2.7\%) (18.1\%)

$888 \quad 60662$

(14.8\%) (18.0\%)

$4774 \quad 76655$

(79.7\%) (22.8\%)

2285188427

(38.2\%) (56.0\%)

$2 \quad 2$

$<0.001$

$2060 \quad 186392$

(34.4\%) (55.4\%)

$1431 \quad 83489$

(23.9\%) (24.8\%)

$1660 \quad 41630$

(27.7\%) (12.4\%)

(14.0\%) (7.4\%)

$<0.001$

$3651 \quad 113851$

(61.0\%) (33.8\%)

2337222695

(39.0\%) (66.2\%)

$\begin{array}{cc}14 & 24 \\ (4-38) & (8-60)\end{array}$

3698269350

(61.8\%) (80.0\%)

195964060

(32.7\%) (19.0\%)

$321 \quad 3089$

$\begin{array}{cc}10 & 47 \\ (0.2 \%) & (<0.1 \%)\end{array}$

$4688 \quad 287256$

(78.3\%) (85.4\%)

$984 \quad 42842$

(16.4\%) (12.7\%)

$208 \quad 4115$

(3.5\%) (1.2\%)

$108 \quad 2333$

(1.8\%) (0.7\%)

0.14

< 0.001
2010-11

Physical

\begin{tabular}{|c|c|c|c|c|c|}
\hline Mental & Physical & $P$ & Mental & Physical & $P$ \\
\hline $\begin{array}{c}8503 \\
(2.3 \%)\end{array}$ & $\begin{array}{l}355722 \\
(97.7 \%)\end{array}$ & & $\begin{array}{c}8726 \\
(2.2 \%)\end{array}$ & $\begin{array}{l}381667 \\
(97.8 \%)\end{array}$ & \\
\hline
\end{tabular}

6622347508

(1.9\%) (98.1\%)

(2.3\%) (97.7\%)

0.004

$\begin{array}{cc}4602 & 230625 \\ (2.0 \%) & (98.0 \%) \\ 2020 & 116883 \\ (1.7 \%) & (98.3 \%)\end{array}$

144119

(2.5\%) (41.5\%)

$171 \quad 63461$

(2.6\%) (18.3\%)

$1038 \quad 60674$

(15.7\%) (17.5\%)

$5248 \quad 79254$

(79.3\%) (22.8\%)

$2679 \quad 192815$

(40.5\%) (55.5\%)

$\begin{array}{cc}3 & 2 \\ (2-4) & (2-4)\end{array}$

$$
<0.001
$$

$<0.001$

$\begin{array}{cc}2316 & 193128 \\ (35.0 \%) & (55.6 \%)\end{array}$

$1574 \quad 85291$

(23.8\%) (24.5\%)

$1814 \quad 42928$

(27.4\%) (12.4\%)

$918 \quad 26161$

(13.9\%) (7.5\%)

\section{$4086 \quad 124756$}

$\begin{array}{cc}4086 & 124756 \\ (61.7 \%) & (35.9 \%)\end{array}$

$2536 \quad 222752$

(38.3\%) (64.1\%)

$18 \quad 25$

$(6-48) \quad(9-62)$

$$
<0.001
$$

$3875 \quad 268725$

(58.5\%) (77.3\%)

238474188

(36.0\%) (21.3\%)

$360 \quad 4550$

(5.4\%) (1.3\%)

$\begin{array}{cc}3 & 45 \\ (<0.1 \%) & (<0.1 \%)\end{array}$

$(<0.1 \%) \quad(<0.1 \%)$

$$
<0.001
$$

$5108 \quad 291146$

(77.1\%) (83.8\%)

$1109 \quad 47356$

(16.7\%) (13.6\%)

$255 \quad 4745$

(3.9\%) (1.4\%)

$147 \quad 3853$

(2.2\%) (1.1\%)
$<0.001$

$<0.001$

$\begin{array}{cc}5935 & 239199 \\ (2.4 \%) & (97.6 \%) \\ 2568 & 116523\end{array}$

(2.2\%) (97.8\%)

$<0.001$

$<0.001$

$\begin{array}{cc}138 & 148847 \\ (1.6 \%) & (41.8 \%)\end{array}$

$200 \quad 66221$

(2.4\%) (18.6\%)

$1463 \quad 61589$

(17.2\%) (17.3\%)

670279065

(78.8\%) (22.2\%)

$2971 \quad 195591$

(34.9\%) (55.0\%)

$\begin{array}{cc}3 & 2 \\ (2-4) & (2-4)\end{array}$

$3202 \quad 196384$

(37.7\%) (55.2\%)

$2019 \quad 89782$

(23.7\%) (25.2\%)

$2261 \quad 43260$

(26.6\%) (12.2\%)

$1021 \quad 26296$

(12.0\%) (7.4\%)

$<0.001$

$5444 \quad 132514$

(64.0\%) (37.3\%)

3059223208

$(36.0 \%) \quad(62.7 \%)$

$\begin{array}{cc}19 & 24 \\ (7-47) & (9-59)\end{array}$

$<0.001$

$5055 \quad 278188$

(59.4\%) (78.2\%)

$2999 \quad 72991$

(35.3\%) (20.5\%)

$443 \quad 4524$

(5.2\%) (1.3\%)

$\begin{array}{cc}6 & 19 \\ (0.1 \%) & (<0.1 \%)\end{array}$

$<0.001$

$$
<0.001
$$

$6378 \quad 292288$

(75) (82.2\%)

$1590 \quad 53699$

(18.7\%) (15.1\%)

$384 \quad 4865$

(4.5\%) (1.4\%)

$144 \quad 4350$

(1.7\%) (1.2\%)
(2.3\%) (97.7\%)

$2487 \quad 114281$

(2.1\%) (97.9\%)

$143 \quad 163966$

(1.6\%) (43.0\%)

$257 \quad 74696$

(2.9\%) (19.6\%)

$1617 \quad 65528$

(18.5\%) (17.2\%)

$6709 \quad 77477$

(76.9\%) (20.3\%)

$3008 \quad 210042$

(34.5\%) (55.0\%)

33

$<0.001$

$3468 \quad 208241$

(39.7\%) (54.6\%)

$2150 \quad 97296$

(24.6\%) (25.5\%)

$2167 \quad 47794$

(24.8\%) (12.5\%)

$941 \quad 28336$

(10.8\%) (7.4\%)

$<0.001$

$5788 \quad 151642$

(66.3\%) (39.7\%)

2938230025

(33.7\%) (60.3\%)

$17 \quad 21$

$(6-39) \quad(9-51)$

$<0.001$

$5706 \quad 314585$

(65.4\%) (82.4\%)

262363069

(30.1\%) (16.5\%)

$390 \quad 4005$

(4.5\%) (1.0\%)

$\begin{array}{cc}7 & 8 \\ (0.1 \%) & (<0.1 \%)\end{array}$

$6199 \quad 301681$

(71.0\%) (79.0\%)

205570614

(23.6\%) (18.5\%)

$315 \quad 4390$

(3.6\%) (1.2\%)

$153 \quad 4447$

$(1.8 \%) \quad(1.2 \%)$
$6239 \quad 267386$

$<0.001$ 
3 Population rates of presentations to Victorian emergency departments by people aged 0-19 years with mental health problems, 2008-2015*

\begin{tabular}{|c|c|c|c|c|c|c|c|}
\hline \multirow[b]{2}{*}{ ICD-10-AM diagnostic category } & \multicolumn{7}{|c|}{ Presentations per 10000 people aged $0-19$ years } \\
\hline & $2008-09$ & $2009-10$ & $2010-11$ & $2011-12$ & $2012-13$ & $2013-14$ & $2014-15$ \\
\hline Intentional self-harm $^{\dagger}$ & 11 & 10 & 10 & 11 & 14 & 15 & 15 \\
\hline F40-F48 (Neurotic, stress-related) & 8 & 9 & 9 & 10 & 11 & 11 & 11 \\
\hline F30-F39 (Mood) & 5 & 6 & 6 & 8 & 10 & 8 & 9 \\
\hline
\end{tabular}

ICD-10-AM = International Classification of Diseases, revision 10, Australian modification. * Based on number of people in Victoria aged 0-19 years for each financial year. ${ }^{18}$ † No ICD-10-AM diagnostic category.

and behavioural and emotional disorders (F90-F98) together accounted for 21127 presentations $(40.3 \%$ of mental health presentations). The annual number of presentations for neurotic and stress-related disorders (mainly anxiety) increased by $46.1 \%$ during the 7-year period (from 1054 to 1540), for behavioural and emotional disorders (mainly conduct disorder) by $83.1 \%$ (from 473 to 866 ), and for mood disorders (mainly depression) by $91.3 \%$ (658 to 1259) (Box 1).

\section{Patient characteristics associated with mental and physical health presentations during 2014-15}

In 2014-15, 6709 mental health presentations were by 15-19-yearolds $(76.9 \%$ of all mental presentations by people aged $0-19$ years), and $1617(18.5 \%)$ by $10-14$-year-olds. Since $2008-09$, the proportion of presentations by 15-19-year-olds for mental health problems had decreased (from 79.7\%) while the proportion for 10-14-year-olds had increased (from 14.8\%). Most mental health presentations during 2014-15 were by girls (5718,65.5\%), whereas fewer than half of all physical health presentations were by girls (171 625, 45.0\%) (Box 2).

The largest proportion of physical health presentations was for children aged $0-4$ years (163966, 43.0\% of physical health presentations).

Over the 7-year period, the number of children aged 0-9 years in Victoria increased, but there was only a negligible increase in the older age groups in which the number of mental health presentations had increased (data not shown). The numbers of girls and boys in Victoria each increased by $1.07 \%$ per annum over the 7 years, but the proportion of boys who presented to an emergency department with a mental health problem decreased while that of girls increased (Box 2); further, the presentation rates for self-harm, stress-related, mood, and behavioural and emotional disorders each increased markedly over the study period (Box 3).

The proportions of mental and physical health presentations to rural and metropolitan EDs were similar, nor were they influenced by socio-economic status of residence (Box 2). The median time to treatment was slightly lower for children with mental health problems (17 min; interquartile range [IQR], 6-39 $\mathrm{min}$ ) than for those presenting with physical health problems (21 min; IQR, 9-51 $\mathrm{min})$.

\section{Relative burden of mental and physical health presentations during 2014-15}

A greater proportion of mental health presentations (5788 presentations, $66.3 \%$ ) than of physical health presentations
(151 642,39.7\%) were triaged as urgent (triage categories 1-3), and a greater proportion took place after hours (10 pm-2 am: 2167, $24.8 \%$ v $47794,12.5 \%$; 2 am-8 am: $941,10.8 \% ; 28336,7.4 \%)$. Fewer mental than physical health presentations met the NEAT target $(5706,65.4 \%$ v $314585,82.4 \%)$. Children presenting for a mental health problem were more likely to be admitted to hospital than those with physical health problems (2055, 23.6\% v 70 614, 18.5\%). Similar patterns applied in other years (Box 2).

\section{Discussion}

This is the first Australian study to investigate trends in presentations to EDs by children and young adults for mental health problems. The number of children who presented to Victorian public EDs increased between 2008-09 and 2014-15; the number of mental health presentations increased by $46 \%$, that of physical health presentations by $13 \%$. Intentional self-harm and psychoactive substance use were the most frequent reasons for mental health presentations. Stress-related, mood, and behavioural and emotional disorders together accounted for $40 \%$ of mental health presentations, and the numbers of presentations for each of these reasons increased rapidly during the 7-year study period. Children who presented with mental health problems were more likely to be triaged as urgent, to present after business hours, to stay longer in the ED, and to be admitted to hospital than those who presented with physical health problems.

Our findings are similar to results reported in the USA, where the number and proportion of mental health visits to EDs by children aged 10-14 years, including those associated with substance use, increased by $21 \%$ between 2006 and $2011 .{ }^{12}$ Earlier studies also found that mental health presentations by children were associated with longer ED stays ${ }^{15}$ and an increased likelihood of admission to hospital. ${ }^{10,14}$ In contrast to American studies, ${ }^{14}$ we found that the number of ED presentations for mood and stress-related disorders, particularly depression and anxiety, rose rapidly. Data from two surveys indicated that the prevalence of major depression in Australia among 4-17-year-olds increased from $2.1 \%$ in 1998 to $3.2 \%$ in $2013-14,{ }^{22}$ but this does not explain the steep rise in presentations to the ED for mood disorders during our study period. We also found that mental health presentations by children aged 10-14 years comprised an increasing proportion of all presentations by children and adolescents, suggesting that community-based care for these children is inadequate.

Our study had several strengths. While other authors have reported the increasing number of children presenting to Victorian EDs, ${ }^{23,24}$ our data extend this work by differentiating between 
trends in the relative proportions and burdens of mental and physical health presentations. While there were some changes to ICD-10-AM coding during the study period, their impact would have been minimal; we examined broad diagnostic categories rather than individual diagnoses, and commenced analyses during the 2008-09 financial year, when diagnoses related to depression became available. Coding of diagnoses in VEMD data are not independently verified by third party assessors, but their integrity is regularly assessed by an external advisory group.

Our study was limited by the quality of the VEMD data, particularly by inaccuracies in diagnostic coding, as codes are generally entered by busy clinicians with limited training in coding. ${ }^{17}$ Data on presentations to private EDs (around 20\% of Victorian EDs that receive children ${ }^{25}$ ) were not available because private EDs are not required to supply data to the VEMD. In addition, we could not compare the characteristics of presentations to community and paediatric hospitals, as hospital campus coding was applied in the dataset. Investigating these differences is important, as presentation characteristics, hospital resources, and management of paediatric mental health presentations may differ between the two hospital types. Further, the VEMD captures only one diagnosis per presentation, as a result of which some physical health presentations (eg, abdominal pain) by patients with underlying mental health problems (eg, anxiety) were probably excluded from the mental health presentation category. Finally, although attention deficit/hyperactivity disorder is the most common mental health diagnosis in Australian young people, ${ }^{2}$ the VEMD does not include an ICD-10-AM code for this diagnosis.

Mental health disorders in children and adolescents account for an increasing number of presentations to EDs, with particularly large increases in the numbers of presentations for depression and behavioural problems. In the 2013-14 Australian Child and Adolescent Survey of Mental Health and Wellbeing (completed by 6310 caregivers of children aged $4-17$ years, $13.9 \%$ of whom were assessed as having had a mental disorder during the previous 12 months), $39.6 \%$ of those who did not seek help for their children's mental health problems did not know where to obtain help, while $36.4 \%$ were uncertain whether assistance was necessary. ${ }^{22}$ General practitioners were the most common source of professional help, but they typically referred children to specialist services that often involved out-of-pocket costs that caregivers could not afford. All these factors may delay treatment, resulting in crisis presentations to EDs.

Potential solutions include public health campaigns to improve recognition by caregivers of the symptoms of mental health problems in children and awareness of where to seek help. Providing GPs with skills and financial resources for managing social, emotional and behavioural problems during early childhood is also important. While Headspace provides mental health services for those aged 12-25 years, our data suggest that younger children need more help. Hubs of care for younger children should include clinicians who offer not only co-located services, but also outreach support to the community and schools to share their expertise and, ultimately, to reduce the number of children who present to EDs with mental health problems.

Competing interests: No relevant disclosures.

Received 10 May 2017, accepted 12 Oct 2017.

(c) 2018 AMPCo Pty Ltd. Produced with Elsevier B.V. All rights reserved.
1 Erskine HE, Moffitt TE, Copeland WE, et al. A heavy burden on young minds: the global burden of mental and substance use disorders in children and youth. Psychol Med 2015; 45: 1551-1563.

2 Lawrence D, Hafekost J, Johnson SE, et al. Key findings from the second Australian Child and Adolescent Survey of Mental Health and Wellbeing. Aust N Z J Psychiatry 2016; 50: 876-886.

3 Bor W, Najman JM, O'Callaghan GM, et al. Aggression and the development of delinquent behaviour in children (Trends and Issues in Criminal Justice No. 207) Canberra: Australian Institute of Criminology, 2001. https://aic.gov.au/publications/tandi/tandizo7 (viewed Oct 2017).

4 Bosquet M, Egeland B. The development and maintenance of anxiety symptoms from infancy through adolescence in a longitudinal sample. Dev Psychopathol 2006; 18: 517-550.

5 Kessler RC, Berglund P, Demler O, et al. Lifetime prevalence and age-of-onset distributions of DSM-IV disorders in the National Comorbidity Survey Replication. Arch Gen Psychiatry 2005; 62: 593-602.

6 Johnson SE, Lawrence D, Hafekost J, et al. Service use by Australian children for emotional and behavioural problems: findings from the second Australian Child and Adolescent Survey of Mental Health and Wellbeing. Aust N Z J Psychiatry 2016; 50: 887-898.

7 Dolan MA, Fein JA; Committee on Pediatric Emergency Medicine. Pediatric and adolescent mental health emergencies in the emergency medical services system. Pediatrics 2011; 127: el356-el366.

8 Newton AS, Hartling L, Soleimani A, et al. A systematic review of management strategies for children's mental health care in the emergency department: update on evidence and recommendations for clinical practice and research. Emerg Med J 2017; 34: 376-384.

9 Reder S, Quan L. Emergency mental health care for youth in Washington State: qualitative research addressing hospital emergency departments' identification and referral of youth facing mental health issues. Pediatr Emerg Care 2004; 20: 742-748.

10 Starling J, Bridgland K, Rose D. Psychiatric emergencies in children and adolescents: an Emergency Department audit. Australas Psychiatry 2006; 14: 403-407.

11 Stewart C, Spicer M, Babl FE. Caring for adolescents with mental health problems: challenges in the emergency department. J Paediatr Child Health 2006; 42: 726-730.

12 Torio CM, Encinosa W, Berdahl T, et al. Annual report on health care for children and youth in the United States: national estimates of cost, utilization and expenditures for children with mental health conditions. Acad Pediatr 2015; 15: 19-35.

13 Baggoley C, Owler B, Grigg M, et al. Expert panel: review of elective surgery and emergency access targets under the national partnership agreement on improving public hospital services. Report to the Council of Australian Governments. Canberra, 2011. Archived: https://web. archive.org/web/20130511193929/http://www.coag.gov. au/sites/default/files/Expert_Panel_Report\%20D0490. pdf (viewed Jan 2018).

14 Grupp-Phelan J, Harman JS, Kelleher KJ. Trends in mental health and chronic condition visits by children presenting for care at U.S. emergency departments. Public Health Rep 2007; 122: 55-61.
15 Mahajan P, Alpern ER, Grupp-Phelan J, et al. Epidemiology of psychiatric-related visits to emergency departments in a multicenter collaborative research pediatric network. Pediatr Emerg Care 2009; 25: $715-720$.

16 Department of Health and Human Services (Victoria). Victorian emergency minimum dataset (VEMD). https:// www2.health.vic.gov.au/hospitals-and-health-services/ data-reporting/health-data-standards-systems/datacollections/vemd (viewed May 2017)

17 Spillane IM, Krieser D, Dalton S, et al. Limitations to diagnostic coding accuracy in emergency departments: implications for research and audits of care. Emerg Med Australas 2010; 22: 91-92.

18 Australian Bureau of Statistics. 3101.0. Australian demographic statistics, Dec 2016. Table 52: estimated resident population by single year of age, Victoria. http://www.abs.gov.au/AUSSTATS/abs@.nsf/ DetailsPage/3101.0Dec\%202016?OpenDocument (viewed May 2017)

19 National Centre for Classification in Health. The International Statistical Classification of Diseases and Related Health Problems, 10th revision, Australian modification (ICD-10-AM). Seventh edition. Sydney: $\mathrm{NCCH}$, University of Sydney, 2010.

20 Australian Bureau of Statistics. 2033.0.55.001. Census of population and housing: Socio-economic indexes for areas (SEIFA), Australia, 2011. Mar 2013. http://www.abs.gov.au/ausstats/abs@.nsf/Lookup/ 2033.0.55.001main+features100132011 (viewed May 2017).

21 Australian Bureau of Statistics. 2033.0.55.001-Census of population and housing: Socio-economic indexes for areas (SEIFA), Australia, 2011. IRSAD. 
Mar 2013. http://www.abs.gov.au/ausstats/abs@.nsf/ Lookup/2033.0.55.001main+features100042011 (viewed May 2017).

22 Lawrence D, Johnson S, Hafekost J, et al. The mental health of children and adolescents: report on the second Australian Child and Adolescent Survey of Mental Health and Wellbeing. Canberra: Department of Health, 2015. https://www.health.gov.au/internet/main/
publishing.nsf/Content/9DA8CA21306FE6EDCA257E27 00016945/\%24File/child2.pdf (viewed Oct 2017).

23 Freed GL, Gafforini S, Carson N. Age distribution of emergency department presentations in Victoria. Emerg Med Australas 2015; 27: 102-107.

24 Lowthian JA, Curtis AJ, Jolley DJ, et al. Demand at the emergency department front door: 10-year trends in presentations. Med J Aust 2012; 196: 128-132. https://www.mja.com.au/journal/2012/196/2/demandemergency-department-front-door-10-year-trendspresentations

25 Department of Health and Human Services (Victoria). Victorian hospital lists: Victorian hospital locations by hospital name. https://www.healthcollect.vic.gov. au/HospitalLists/MainHospitalList.aspx (viewed May 2017). 